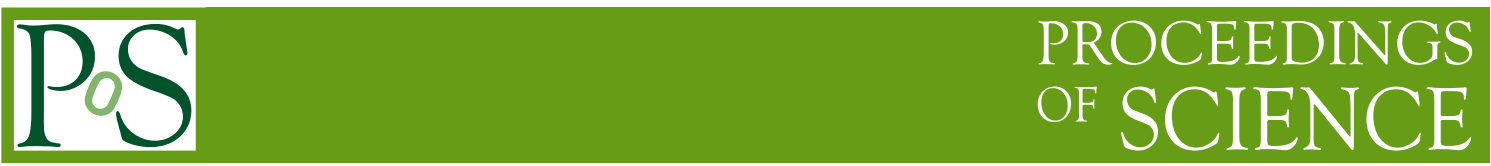

\title{
New results from TopFitter
}

\author{
Stephen Brown, ${ }^{a}$ Andy Buckley, ${ }^{a}$ Christoph Englert, ${ }^{a}$ James Ferrando, ${ }^{b}$ \\ Peter Galler, ${ }^{a, *}$ David Miller, ${ }^{a}$ Chaowaroj Wanotayaroj, ${ }^{b}$ Neil Warrack ${ }^{a}$ and \\ Chris White ${ }^{c}$ \\ ${ }^{a}$ University of Glasgow, \\ University Avenue, Glasgow G12 8QQ, United Kingdom \\ ${ }^{b}$ Deutsches Elektronen-Synchrotron (DESY) \\ Notkestraße 85, Hamburg, Germany \\ ${ }^{c}$ Queen Mary University of London, \\ Mile End Road, London E1 4NS, United Kingdom \\ E-mail: s.brown.7@research.gla.ac.uk, andy.buckley@cern.ch, \\ christoph.englert@glasgow.ac.uk, james.ferrando@desy.de, \\ peter.galler@glasgow.ac.uk, david.j.miller@glasgow.ac.uk, ma.x@cern.ch, \\ neil.warrack@cern.ch, christopher.white@qmul.ac.uk
}

We present updated results for a global fit of dimension-six operators in the top quark sector of the Standard Model Effective Field Theory using experimental data from the Tevatron and the LHC at 7, 8 and $13 \mathrm{TeV}$ center-of-mass energy. We include all contributions from dimension-six operators up to and including $\Lambda^{-4}$ in the effective field theory expansion. Furthermore we take the top quark decay into account and include in the fit experimental measurements which provide results for observables that depend on the kinematics of the top (anti)quark decay products. This opens up the possibility to study additional operators such as four-fermion operators which couple top quarks directly to leptons. A new strategy for sampling the parameter space of Wilson coefficients and treatment of systematic uncertainties is employed. In this contribution we restrict ourselves to present results in terms of bounds on individual operators.

40th International Conference on High Energy physics - ICHEP2020

July 28 - August 6, 2020

Prague, Czech Republic (virtual meeting)

\footnotetext{
${ }^{*}$ Speaker
} 


\section{Introduction}

The Standard Model Effective Field Theory (SMEFT, see Ref. [1] for a recent review) is a wellestablished tool to study new physics beyond the Standard Model (SM) with a large energy scale $\Lambda$ (compared to the electro-weak scale) under very general assumptions. In particular, it can be applied in a bottom-up approach where the UV dynamics is unknown. In this approach the Lagrangian is expanded in terms of $\Lambda^{4-d}$

$$
\mathcal{L}=\mathcal{L}_{\mathrm{SM}}+\sum_{d=5}^{\infty} \frac{1}{\Lambda^{d-4}} \sum_{i} C_{i}^{(d)} O_{i}^{(d)}
$$

where $O_{i}^{(d)}$ are operators of mass dimension $d$ and $C_{i}^{(d)}$ are the corresponding Wilson coefficients. Thus, the low-energy effects of new physics at a high energy scale $\Lambda$ are parametrised in terms of new interactions represented by the operators $O_{i}^{(d)}$. The Wilson coefficients which parametrise the strength of these interactions are free parameters.

TopFITTER uses this bottom-up approach to fit the Wilson coefficients to available experimental data from the Tevatron and the LHC. Specifically, we focus on effective operators and their Wilson coefficients which are relevant for top quark physics, i.e. operators that include at least one top quark field. We expand the Lagrangian up to $\Lambda^{-2}$. Since operators of $d=5$ do not contribute to top quark processes we only include effective operators with $d=6$. Previous results [2] were published using a limited set of operators and data sets. Using a new approach for scanning the parameter space allows us to increase the number of fitted operators considerably. Furthermore, we include experimental data from LHC run II up to an integrated luminosity of $L=139 / \mathrm{fb}$.

We note that effective field theory (EFT) fits in the top quark sector have become quite popular with several groups [3-5] publishing results focussing on different aspects of the data, observables and fit methodology. TopFitTer aims at including a comprehensive data set using most of the available experimental collider data on top quark physics, studying as many effective top operators as possible, investigating the impact of double operator insertions on the fit, including observables at the level of decayed top quarks and using fiducial measurements in the fit. All of these points have been addressed by ToPFitTER. In particular, the last point was presented [6] at the ICHEP 2018, where we showed an example of a fit using fiducial results [7]. However, in this contribution we focus on experimental results at the parton level.

\section{Operators}

We study dimension-six operators which involve at least one top quark field. Under the assumption of a flavour symmetry $\mathrm{U}(2)_{q} \times \mathrm{U}(2)_{u} \times \mathrm{U}(2)_{d}$ in the first two quark generations, assuming lepton universality and lepton flavour conservation and assuming $C P$ conservation in the EFT contributions there are 42 effective operators in the top quark sector of the SMEFT. From this set of operators we include all dipole operators (4) all charged and neutral current operators (4) all heavy-light fourquark operators (14) and all lepton-top operators (8). In addition we include the non-top operator $O_{G}=f^{A B C} G_{\mu}^{A v} G_{v}^{B \rho} G_{\rho}^{C \mu}$ in the fit. This is a total of 31 operators. The operator $O_{G}$ contributes for example to the gluon channel in top-quark pair production. It is well constrained [8,9] from jet physics but it is still interesting to see how much it can be constrained by top quark physics. We adopt the Warsaw basis [10] and summarize the considered operators in Tab. 1. 
Table 1: Operators used in the fit. We use the same notation as [10].

\begin{tabular}{|c|c|c|c|}
\hline category & operators & category & operators \\
\hline dipole & $O_{u B}^{33}, O_{u G}^{33}, O_{u W}^{33}, O_{d W}^{33}$ & $\begin{array}{l}\text { neutral/charged } \\
\text { current }\end{array}$ & $O_{\varphi q}^{(1) 33}, O_{\varphi q}^{(3) 33}, O_{\varphi u}^{33}, O_{\varphi u d}^{33}$ \\
\hline heavy-light & $\begin{array}{c}O_{q q}^{(1) i i 33}, O_{q q}^{(1) i 33 i}, O_{q q}^{(3) i 33}, O_{q q}^{(3) i 33 i}, O_{u u}^{i i 33}, \\
O_{u u}^{i 33 i}, O_{q u}^{(1) i 33}, O_{q u}^{(1) 33 i i}, O_{q u}^{(8) i i 33}, O_{q u}^{(8) 33 i i}, \\
O_{q d}^{(1) 33 i i}, O_{q d}^{(8) 33 i i} O_{u d}^{(1) 33 i i}, O_{u d}^{(8) 33 i i}\end{array}$ & lepton-top & $\begin{array}{c}O_{\ell q}^{(1) i i 33}, O_{\ell q}^{(3) i i 33}, O_{\ell u}^{i i 33}, O_{\text {eu }}^{i i 33} \\
O_{q e}^{33 i i}, O_{\text {ledq }}^{i i 33}, O_{\text {lequ }}^{(1) i i 3}, O_{\text {lequ }}^{(3) i 33}\end{array}$ \\
\hline
\end{tabular}

\section{Experimental input}

We include as many experimental results as possible using Tevatron as well as LHC run I and II data. Here we do not list all the experimental analyses explicitly but provide in Tab. 2 an overview of the considered processes and number of degrees of freedom. The processes are denoted as follows:

Table 2: Effective number of degrees of freedom per process and collaboration. The total number is 831 .

\begin{tabular}{lcccccccccccc}
\hline collaboration & $t \bar{t}$ & $t \bar{t}(\ell \ell)$ & $t \bar{t}(\ell j)$ & $t \bar{t} W$ & $t \bar{t} Z$ & $t \bar{t} Z(\ell \ell)$ & $t j$ & $t j(\ell)$ & $t b$ & $t W$ & $t j Z$ & $t j Z(\ell \ell)$ \\
\hline ATLAS & 57 & 29 & & 2 & 2 & & 22 & 8 & & 1 & 1 & 1 \\
CMS & 205 & 407 & & 2 & 2 & 7 & 6 & 10 & & 1 & & 1 \\
ATLAS \& CMS & 1 & & & & & & & & 1 & 2 & & \\
CDF & 20 & & & & & & 1 & & & & & \\
D0 & 21 & 7 & 3 & & & & 1 & & & & & \\
CDF \& D0 & 6 & 1 & 1 & & & & 1 & & 1 & & & \\
\hline
\end{tabular}

top-quark pair production $(t \bar{t})$, top-quark pair production with (semi-)leptonic decay $(t \bar{t}(\ell \ell), t \bar{t}(\ell j))$, top-quark pair production in association with a vector boson $(t \bar{t} W, t \bar{t} Z)$, including leptonic $Z$ decay $(t \bar{t} Z(\ell \ell))$, single top $t$-channel, $s$-channel and associated production $(t j, t b, t W), t$-channel production including leptonic top decay $(t j(\ell))$, single top production in association with a $Z$ boson $(t j Z)$ including leptonic $Z$ decay $(t j Z(\ell \ell))$. The degrees of freedom count the effective number of observables for each process, e.g. for a normalised differential cross section the effective number of degrees of freedom is $N_{\text {bins }}-1$ where $N_{\text {bins }}$ is the number of bins. In principle all of these observables are used for the fit. However, due to the fact that correlation between different distributions measured in the same analysis or even bin-to-bin correlations are often not provided we can only select a subset of these observables to avoid double counting. Details about the procedure that selects the appropriate subset is given in Sec. 6. Even though the TopFitTer frame work is able to handle fiducial analyses [6] we restrict ourselves to parton-level analyses for now.

\section{Theoretical input}

We generate the SM and EFT contributions to all processes with MADGRAPH5_AMC@NLO v.2.6.6 [11]. The SM contribution is generated at next-to-leading order (NLO) QCD. The EFT contributions are generated at leading order (LO) QCD using the UFO [12] model file SMEFTsim [13]. Cross sections can be decomposed as follows

$$
\sigma=\sigma_{\mathrm{SM}}+\frac{C_{i}}{\Lambda^{2}} \sigma_{i}^{(1)}+\frac{C_{i} C_{j}}{\Lambda^{4}} \sigma_{i j}^{(2)},
$$


where $\sigma$ denotes a cross section or the bin of a differential cross section. $\sigma_{\mathrm{SM}}$ is the SM contribution, $\sigma_{i}^{(1)}$ are the contributions from the interference of the EFT contribution with the SM contribution and $\sigma_{i j}^{(2)}$ consists of EFT squared contributions $(i=j)$ and cross terms $(i \neq j)$ where in both cases single as well as double operator insertions can contribute. MAdGRAPH5_AMC@NLO allows the calculation of all contributions $\sigma_{i}^{(1)}$ and $\sigma_{i j}^{(2)}$ separately such that they can be stored and used to reconstruct the observable for any point in parameter space using Eq. (1). This is a huge advantage with respect to the previous interpolation approach [2]. Observables that are more complicated than cross sections, e.g. asymmetries, are constructed from cross section observables that can be calculated via Eq. (1). We also generate predictions for processes with top quark decay in order to be able to include observables at the level of the top quark decay products. However, top quark decay breaks the simple structure given in Eq. (1). Therefore we need to treat processes with top decay differently. To this end we apply the narrow width approximation to the top quark decay and calculate the decay at LO QCD using MADSPIN $[14,15]$ such that

$$
\sigma\left(p p \rightarrow t \bar{t} \rightarrow b \bar{b} \ell^{+} \ell^{-} v_{\ell} \bar{v}_{\ell}\right) \equiv \frac{\tilde{\sigma}}{\Gamma_{t}^{2}}
$$

where $\tilde{\sigma}$ is the cross section where the top width $\Gamma_{t}$ was factored out. The numerator has the same structure of a cross section without decay given in Eq. (1) and the denominator has as similar polynomial structure

$$
\Gamma=\Gamma_{\mathrm{SM}}+\frac{C_{i}}{\Lambda^{2}} \Gamma_{i}^{(1)}+\frac{C_{i} C_{j}}{\Lambda^{4}} \Gamma_{i j}^{(2)} .
$$

Hence, we can calculate the numerator and denominator in Eq. (2) separately and combine them. In particular, we need to calculate the SM and EFT contributions to numerator and denominator only once.

\section{Uncertainties}

In addition to the experimental uncertainties which are given by the results of the analyses we have to take into account the theoretical uncertainties due to renormalisation and factorisation scale $\left(\mu_{R}, \mu_{F}\right)$ variations and parton distribution function (PDF) as well as $\alpha_{s}$ uncertainties. To this end we calculate $\mu_{R}, \mu_{F}$, PDF and $\alpha_{s}$ variations for all SM and EFT contribution to all observables. Specifically, we vary $\mu_{R}, \mu_{F}=\left\{m_{t} / 2, m_{t}, 2 m_{t}\right\}$ at the central PDF set member and take the envelope of this variation as the theoretical error associated with the scale uncertainty. In addition we vary the PDF member and values for $\alpha_{s}$ using the PDF set PDF4LHC15_nlo_30_pdfas [16] at $\mu_{R}=\mu_{F}=m_{t}$. The theoretical PDF and $\alpha_{s}$ uncertainties are determined according to the PDF4LHC recommendations [16]. Scale, PDF and $\alpha_{s}$ uncertainties are added in quadrature to obtain the total theoretical uncertainty for each observable. Finally, the theoretical uncertainty and the experimental uncertainty are added in quadrature to obtain the total uncertainty for each observable.

\section{Fit}

The fit is based on the $\Delta \chi^{2}$ constructed from the experimental results and the theoretical predictions as a function of the Wilson coefficients. We use the correlation or covariance information provided by the experimental analyses. However, not all analyses provide covariance information between different simultaneously measured distributions. Hence, in such cases we include only one of the measured distributions in the $\Delta \chi^{2}$ to avoid double counting. In cases where bin-to-bin covariance information is also missing we select only a single bin from a distribution. Furthermore, we 
assume that correlations between analyses from different collaborations or for different collider energies and final states are uncorrelated. The results presented in the following were obtained using a dynamical method of choosing the distribution or bin among all potentially correlated distributions. In this approach the most sensitive observables are selected which may vary from parameter point to parameter point. Hence, we potentially use all available observables but among those without sufficient correlation information we select the most sensitive one depending on the point in parameter space. In this contribution we provide $95 \%$ confidence level (CL) constraints on individual Wilson coefficients by numerically solving

$$
1-C L>\int_{\Delta \chi^{2}(\mathbf{C})}^{\infty} d x f_{\chi^{2}}(x, k)
$$

for the Wilson coefficients $\mathbf{C}$, where $f_{\mathcal{\chi}^{2}}$ is the cumulative distribution function of the $\chi^{2}$ distribution and $k=N_{\text {param }}$ is the number of degrees of freedom which is equal to the number of Wilson coefficients, i.e. for constraints on individual operators $k=1$.

The results are presented in Fig. 1 which shows the 95\% CL constraints on individual Wilson coefficients, i.e. for each Wilson coefficient the fit was performed with all other Wilson coefficients set to zero. The plot on the left-hand side shows the constraints for non-lepton operators. Here we compare the results when including only the SM-EFT interference terms (linear) with the case where also the squared contributions are taken into account (quadratic). The comparison between these two cases allows us to get an estimate of the robustness of the results with respect to contributions from higher orders in the EFT expansion, i.e. operators of higher mass dimension. For those Wilson coefficients where these two results are similar we expect a good convergence of the series. We also see that the quadratic contributions can improve as well as weaken the bounds. Furthermore, we see that the four-quark operators are constrained quite well (when including quadratic contributions). This can be further improved by including $t \bar{t} t \bar{t}$ and $t \bar{t} b \bar{b}$ production in the fit. The Wilson coefficients $C_{\varphi q}^{(1) 33}$, $C_{\varphi u}^{33}$ and $C_{u B}$ which contribute to the $t-Z$ and $t-\gamma$ couplings are not that well constrained. This can be improved by including $t \bar{t} \gamma$ associated production in the fit. However, as Tab. 2 shows most of the observables are measured in the $t \bar{t}$ and single-top production. An increased experimental effort in measuring the top couplings to the $Z$ and $\gamma$ would be desirable. The right-hand side of Fig. 1 shows the constraints for the top-lepton operators. All Wilson coefficients except $C_{\text {lequ }}^{(3) i i 33}$ are basically unconstrained by the experimental data already at the individual level taking quadratic contributions into account.

Preliminary studies show that $C_{\ell \text { equ }}^{(3) i i 33}$ is also strongly affected by double operator insertions. Specifically, the constraints are weaker when only single insertions are allowed. This is an effect which deserves further study. However, the effect of double operator insertions on the other Wilson coefficients is small when only individual operators are fitted. The effects are expected to increase for marginalised bounds where double insertions of two different operators can occur.

\section{Conclusion}

In this contribution we present $95 \% \mathrm{CL}$ constraints for 31 operators in the top quark sector of the SMEFT at the individual level. This was achieved using the TopFitTer frame work which is briefly described. Unfortunately, 7 of the 8 top-lepton operators cannot be constrained given the current experimental data, precision and observables. Dedicated studies are necessary to investigate if this situation can be improved with new processes involving leptons and top quarks and/or new observables and multivariate analyses. The results also show weaker constraints for operators 

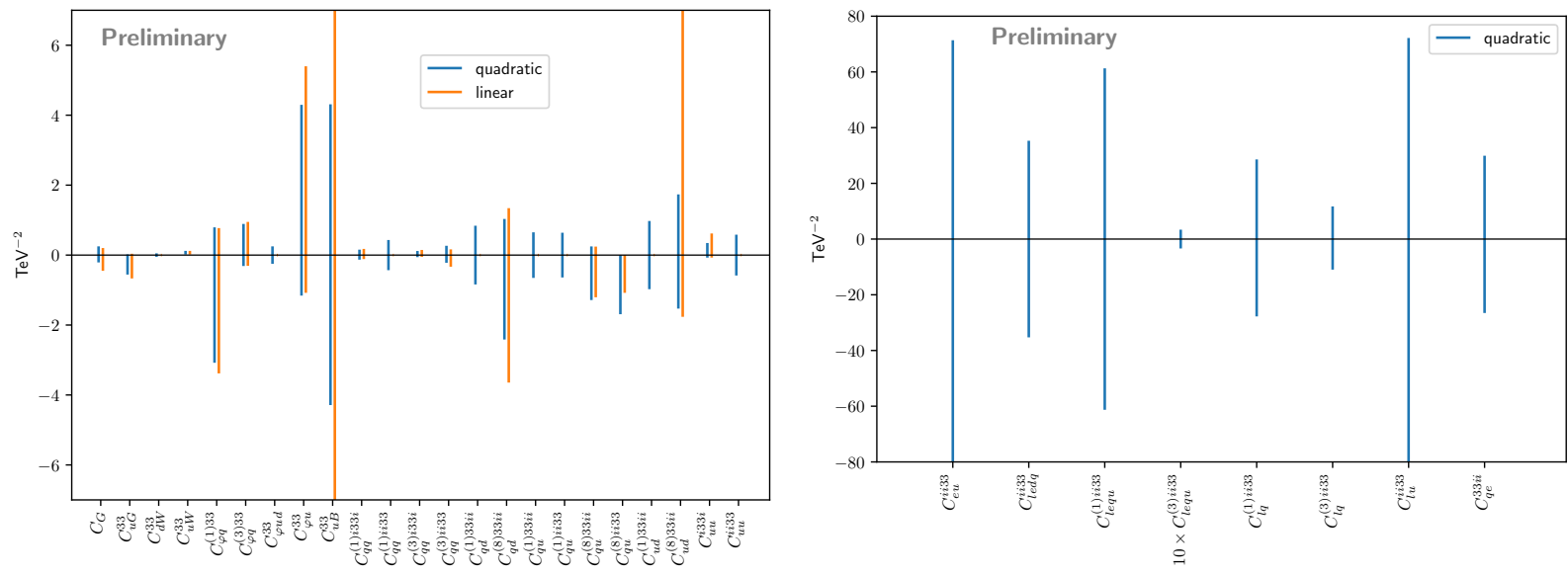

Figure 1: Constraints on individual non-lepton (left) and top-lepton (right) Wilson coefficients at 95\% CL.

related to the $t-Z$ and $t-\gamma$ couplings. Underrepresentation of relevant processes in past analyses could be one reason.

A dedicated publication of the results presented here and marginalised constraints as well as detailed studies of the impact of double operator insertions, top quark decay and different observables on the fit is in progress. A first application of the updated TopFitTER was presented in Ref. [17] where it was used to obtain constraints on a composite Higgs model.

\section{References}

[1] I. Brivio and M. Trott, Phys. Rept. 793 (2019) 1-98, arXiv: 1706.08945 [hep-ph].

[2] A. Buckley, C. Englert, J. Ferrando, D. J. Miller, L. Moore, M. Russell, and C. D. White, JHEP 04 (2016) 015, arXiv: 1512.03360 [hep-ph].

[3] N. P. Hartland, F. Maltoni, E. R. Nocera, J. Rojo, E. Slade, E. Vryonidou, and C. Zhang, JHEP 04 (2019) 100, arXiv: 1901.05965 [hep-ph].

[4] G. Durieux, A. Irles, V. Miralles, A. Peñuelas, R. Pöschl, M. Perelló, and M. Vos, JHEP 12 (2019) 098, arXiv: 1907.10619 [hep-ph].

[5] I. Brivio, S. Bruggisser, F. Maltoni, R. Moutafis, T. Plehn, E. Vryonidou, S. Westhoff, and C. Zhang, JHEP 02 (2020) 131, arXiv: 1910.03606 [hep-ph].

[6] S. Brown, A. Buckley, C. Englert, J. Ferrando, P. Galler, D. J. Miller, L. Moore, M. Russell, C. White, and N. Warrack, PoS ICHEP2018 (2019) 293, arXiv: 1901.03164 [hep-ph].

[7] ATLAS Collaboration, M. Aaboud et al., Phys. Rev. D 98 no. 1, (2018) 012003, arXiv: 1801.02052 [hep-ex].

[8] F. Krauss, S. Kuttimalai, and T. Plehn, Phys. Rev. D 95 no. 3, (2017) 035024, arXiv: 1611.00767 [hep-ph].

[9] V. Hirschi, F. Maltoni, I. Tsinikos, and E. Vryonidou, JHEP 07 (2018) 093, arXiv: 1806.04696 [hep-ph].

[10] B. Grzadkowski, M. Iskrzynski, M. Misiak, and J. Rosiek, JHEP 10 (2010) 085, arXiv: 1008.4884 [hep-ph].

[11] J. Alwall, R. Frederix, S. Frixione, V. Hirschi, F. Maltoni, O. Mattelaer, H. S. Shao, T. Stelzer, P. Torrielli, and M. Zaro, JHEP 07 (2014) 079, arXiv: 1405.0301 [hep-ph].

[12] C. Degrande, C. Duhr, B. Fuks, D. Grellscheid, O. Mattelaer, and T. Reiter, Comput. Phys. Commun. 183 (2012) 1201-1214, arXiv: 1108.2040 [hep-ph].

[13] I. Brivio, Y. Jiang, and M. Trott, JHEP 12 (2017) 070, arXiv: 1709.06492 [hep-ph].

[14] S. Frixione, E. Laenen, P. Motylinski, and B. R. Webber, JHEP 04 (2007) 081, arXiv: hep-ph/0702198.

[15] P. Artoisenet, R. Frederix, O. Mattelaer, and R. Rietkerk, JHEP 03 (2013) 015, arXiv: 1212 . 3460 [hep-ph].

[16] J. Butterworth et al., J. Phys. G 43 (2016) 023001, arXiv: 1510.03865 [hep-ph].

[17] S. Brown, C. Englert, P. Galler, and P. Stylianou, Phys. Rev. D 102 no. 7, (2020) 075021, arXiv: 2006.09112 [hep-ph]. 